\title{
Study on acoustic and aerodynamic performance of reactive muffler
}

\author{
Zhenyu Deng ${ }^{1, a}$, Junchuan Niu ${ }^{1, b}$, Fusheng Sui ${ }^{2, c}$ \\ ${ }^{1}$ School of Mechanical Engineering, Shandong University, Jinan 250061, China; \\ ${ }^{2}$ The Key Laboratory of Noise and Vibration Research, Institute of Acoustics, Chinese Academic of \\ Sciences, Beijing 100190, China. \\ azydengsdu@sina.cn, bniujc@sdu.edu.cn, csui@mail.ioa.ac.cn
}

Keywords: Transmission loss, Pressure loss, Oblique inlet tube, Insert tube.

\begin{abstract}
In order to get a further reduction on pressure loss of a reactive muffler, the interior flow field of the muffler and the generation mechanism of pressure loss are investigated by the CFD method. Then, the influences of the insert tube length on the acoustic and aerodynamic performance of the expansion chamfer muffle are discussed. Results show that the spatial location of expansion or compression vents are so extremely important for the acoustic modes that one can adjust their locations to promote the acoustic and aerodynamic performance of the reactive muffler.
\end{abstract}

\section{Introduction}

Acoustic modes of chamber have a great impact on acoustic attenuation characteristics. Ih [1] and Selamet [2] analyzed the higher-order acoustic modes in the muffler to show how the locations of inlet and outlet tubes affect the acoustic attenuation characteristics of mufflers. Fang [3] studied the three-dimensional acoustic modes of the reactive muffler and showed that placing the inlet/outlet tube on the nodal area of acoustic modes can effectively improve the acoustic performance. Chu [4] applied an arc transition on the outlet tube of the expansion chamber muffler to lower pressure loss which is brought by the offsetting outlet tube, but the pressure loss was still relatively high in comparison with the original concentric muffler. Therefore, in this paper, an oblique inlet tube has been introduced to further decrease the pressure loss. Then, the influences of the oblique insert tube on the performance of the expansion chamber muffler are discussed in detail.

\section{Theoretical basis for calculation}

The three-dimensional sound propagation in a static and inviscid fluid condition is governed by the Helmholtz equation [5]

$$
\nabla^{2} p-\frac{1}{c_{0}^{2}} \frac{D^{2} p}{D t^{2}}=0
$$

where $\nabla^{2}$ is the Laplace operator, $p$ is the sound pressure, and $C_{0}$ is the sound speed. Discrete and solve Eq.(1) with the acoustic boundary conditions by FEM. The equation is written as

$$
\left([\mathbf{K}]+\frac{i \rho_{0} \omega}{z_{n}}[\mathbf{C}]-k_{0}^{2}[\mathbf{M}]\right)\left\{p_{i}\right\}=-i \rho_{0} \omega \mu_{n}\{\mathbf{F}\}
$$

where $\mathbf{M}, \mathbf{C}, \mathbf{K}$, and $\mathbf{F}$ are the mass, damping stiffness matrices and load vector, respectively.

The evaluating index for acoustic performance is transmission loss (TL), which is defined by the subtraction of acoustic power level between inlet and outlet of the muffler as

$$
T L=10 \log \frac{W_{i}}{W_{t}}=10 \log \frac{p_{i}^{2} S_{1}}{p_{t}^{2} S_{2}}=20 \log \frac{p_{i}}{p_{t}}
$$

The aerodynamic performance is evaluated by pressure loss generally. Pressure loss may be obtained from the interior flow field, calculated by CFD method which is based on continuity equation and Reynolds Average Navier-Stokes equations (RANS) 


\section{The impact of oblique inlet tube}

The offset outlet method based on the suppression of acoustic modes increases pressure loss, though it improves acoustic performance [4]. Paper [4] use the arc transition on the compression vent to lower pressure loss but works not good enough. We apply an oblique inlet tube to solve this problem. Set the concentric simple expansion chamber muffler as the original model, offset outlet tube one as model A. These two model are from paper [4]. The one both with offset outlet tube and oblique inlet tube is model B. Figure 1 illustrates the geometries of model A and model B. The chamber diameter $D_{1}=200 \mathrm{~mm}$, the chamber length $L_{1}=200 \mathrm{~mm}$, the radius of transitional arc is $\mathrm{R}_{1}=10 \mathrm{~mm}$, the diameters of inlet tube and outlet tube are both $\mathrm{d}_{1}=36 \mathrm{~mm} . \mathrm{O}_{1}, \mathrm{O}_{2}$ are the centers of expansion vent and compression vent respectively, $\mathrm{S}_{2}=63 \mathrm{~mm}$ is the offset distance, $\theta_{S}$ is the oblique angle.

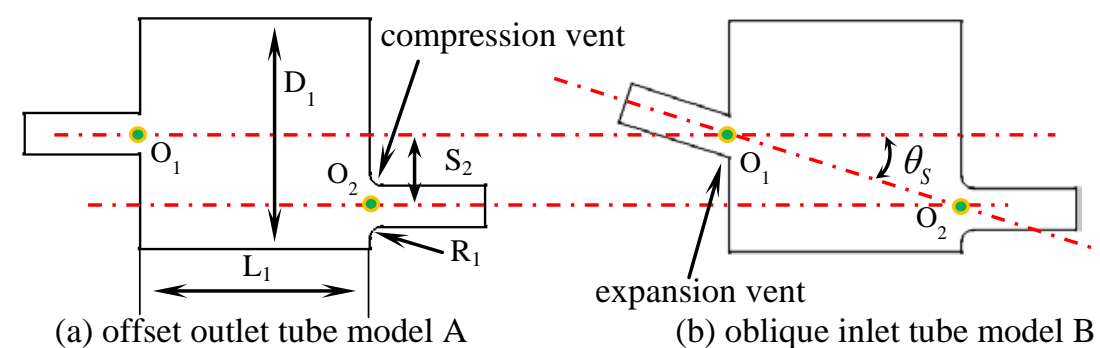

Figure 1. Geometries of model A and model B

Calculate the interior steady flow field and the acoustic field of three models to obtain the pressure loss and TL by commercial software Fluent and Virtual.Lab respectively. Figure 2(a) shows that the pressure loss curve of model B almost overlaps the curve of the original concentric model. That means the oblique inlet tube scheme can effectively get a further reduction on pressure loss. And in Figure 2(b), both model A and model B get a better acoustic performance than the original concentric model and they show little difference as to TL curve. Hence, We concluded that oblique inlet tube can effectively decrease the pressure loss without impacting TL.

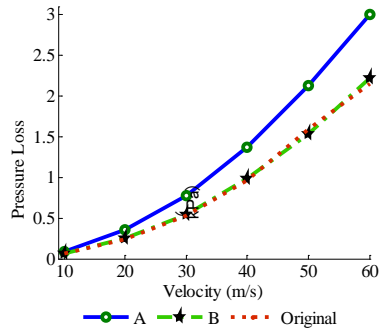

(a) Pressure loss

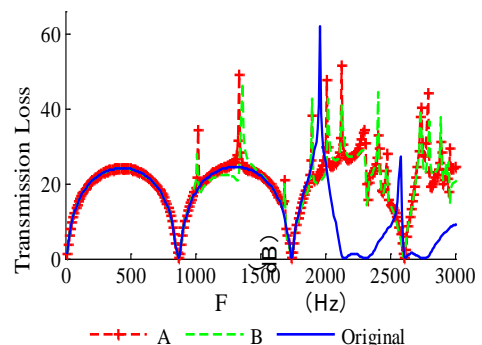

(a) Transmission loss

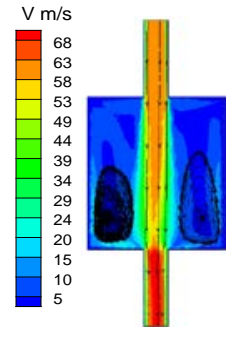

(a) Original

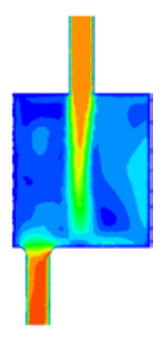

(b) $\mathrm{A}$

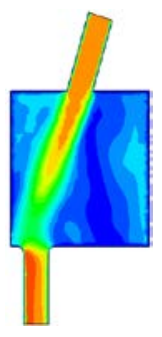

(c) B

Figure 2. Pressure loss and transmission loss

Figure 3. Contour of velocity

Figure 3 shows the contour of velocity on the same cross section of these three models when the inlet velocity is set as $60 \mathrm{~m} / \mathrm{s}$. The black flow traces in Figure 3(a) shows vortex in chamber. The strong friction between the entering high speed airflow and low speed vortex brings much pressure loss, being the first cause for pressure loss. The velocity of entering airflow forcedly altered by the block of chamber in Figure 3(b), consuming much kinetic energy. Oblique inlet tube in Figure 3(c) guides the entering airflow to a better direction and avoid the altering of velocity. Kinetic energy consuming is the second cause, also the reason for higher pressure loss in Figure 3(b). The third is the compression of airflow at compression vent. Oblique inlet tube and arc transition are respectively using the second and third cause of pressure loss mechanism. Insert tube may take good use of the first cause to lower pressure loss.

\section{Research on oblique insert tube}

A new model $\mathrm{C}$ with oblique inlet insert tube is built upon model $\mathrm{B}$ to study how the oblique insert tube affect the performance of the muffler. Figure 4(a) shows the structure of model C. Where $\mathrm{L}_{C}$ is 
the length of insert tube, the wall thickness of insert tube is $1 \mathrm{~mm} . \mathrm{L}_{\mathrm{C}}$ ranges from $0 \mathrm{~mm}$ to $180 \mathrm{~mm}$. The increment is $20 \mathrm{~mm}$. Calculate the pressure loss in every length of insert tube at the speed of 60 $\mathrm{m} / \mathrm{s}$, followed by the corresponding transmission loss.

Figure 4(b) shows the pressure loss with different length of insert tube. Pressure loss decreases sharply and more effectively when the insert tube is relatively shorter. And it decreases more slowly but steadily when the insert tube gets longer. Figure 4(c) shows that the use of insert tube separates the entering airflow from vortex and shorten their interactive friction path, leading to a lower pressure loss. Application of insert tube is a measure taken from the first cause of pressure loss mechanism.

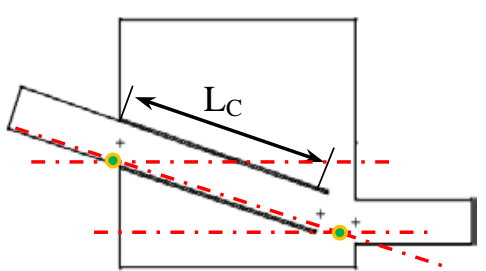

(a) Geometry of model C

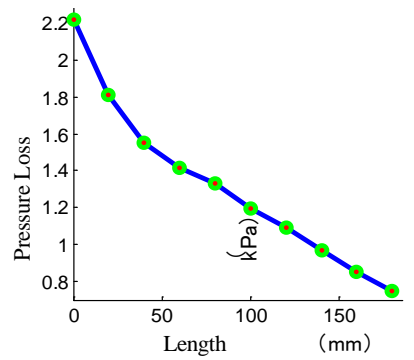

(b) Pressure loss at different length

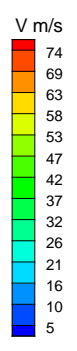

$\mathrm{L}_{\mathrm{C}}=20 \mathrm{~mm} \quad \mathrm{~L}_{\mathrm{C}}=120 \mathrm{~mm}$

(c) Contour of velocity

Figure 4. Structure, pressure loss curve of model $C$ and contour of velocity at different $\mathrm{L}_{\mathrm{C}}$

Figure 5 is the TL curve at different $L_{C}$. In Figure 5(a), the sharp peaks on TL curve when $L_{C}$ is 0 $\mathrm{mm}$ are due to the acoustic modes suppressed by the location of inlet tube [4]. Two obvious resonant peaks brought by insert tube arise when $\mathrm{L}_{C}$ is $20 \mathrm{~mm}$. And the suppression to acoustic modes gets weaker, leading to a smaller TL and anti-resonance trench in most modal frequency, when insert tube gets longer. In Figure 5(b), a resonant peak moves to lower frequency as $L_{C}$ gets much bigger. And the bigger $\mathrm{L}_{C}$ leads to a better low-frequency but a worse middle and high frequency acoustic attenuation performance, especially when $\mathrm{L}_{\mathrm{C}}$ is $180 \mathrm{~mm}$. Many acoustic modes are excited in 180 mm curve subsequently leading to many zero points on TL curve, which is the cause for the worse middle and high frequency acoustic attenuation performance. Model C doesn't change the location of inlet tube except for that of expansion vent. And model $\mathrm{C}$ with a long insert tube excites most of the acoustic modes. Hence, We conclude that the location of expansion vent is the key to the state of excitation or suppression of acoustic modes.

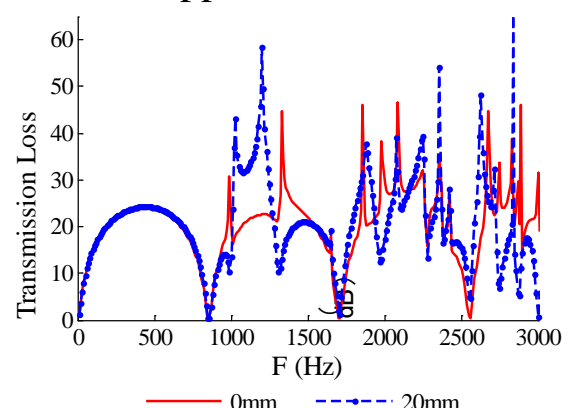

(a)

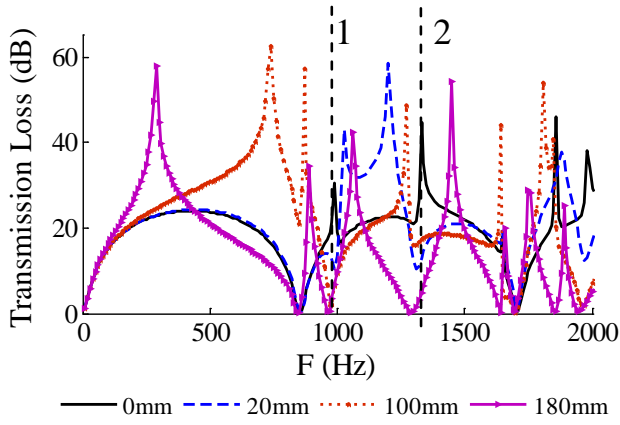

(b)

Figure 5. Transmission loss of model $\mathrm{C}$ at different $\mathrm{L}_{\mathrm{C}}$

In order to validate the impact to acoustic modes caused by the location of expansion vent, build the model $D$ with inlet insert tube offseted by distance of $S_{2}$ in Figure 6(a). And make sure the center of the expansion vent is on the symmetric axis of chamber. Thus the centers of expansion vent of model D and model B are on the same horizontal symmetric axis. Figure 6(b) TL curves of these two models. We can see that the offset of inlet tube changes little of the state of excitation or suppression of acoustic modes. The TL curves of these two models are quite close except for the impact of resonant peaks. Model D gets a better low frequency acoustic attenuation performance. And the insert tube shorten the friction path of entering airflow which can decrease pressure loss. Finally, comparing model D with original model, result shows that both of the acoustic and aerodynamic performance are promoted by the use of oblique inlet insert tube and offset outlet tube. 


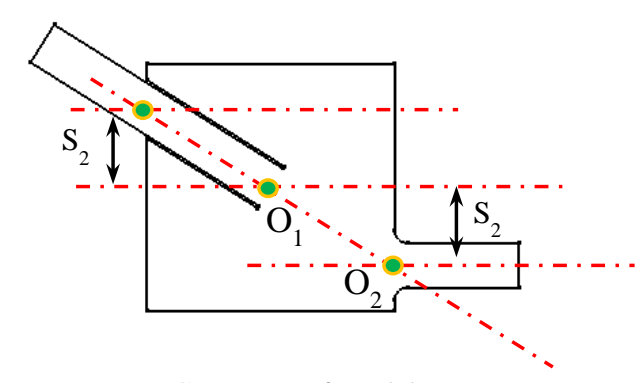

(a) Geometry of model D

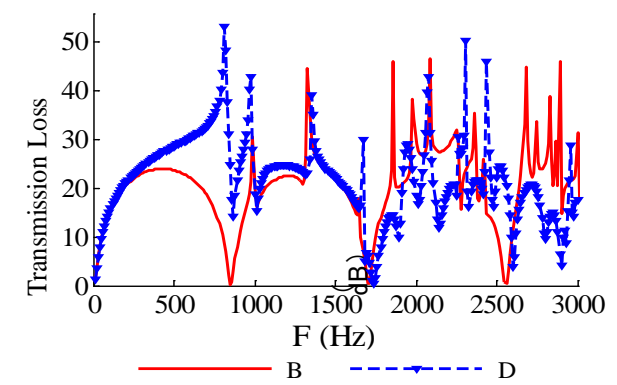

(b) Pressure loss of model B and model D

Figure 6. Comparison of model B and model D

\section{Summary}

The mechanism of pressure loss generation in the chamber has been investigated through analyzing the interior flow field. And we conclude that interactive friction between entering flow and vortex, altering of velocity, and compression of airflow are the three vital factors for pressure loss generation. The reduction of pressure loss after applying oblique inlet tube and oblique insert tube proves this conclusion. And measures can be taken according to this mechanism to lower pressure loss of other reactive mufflers.

The spatial locations of the expansion vent and compression vent are essentially the key to the state of excitation or suppression of acoustic modes which impact acoustic performance a lot. We can adjust the oblique angle of inlet or outlet tube and length of insert tube to both promote the acoustic and aerodynamic performance of the reactive muffler in the condition that the expansion vent and compression vent are right located at the nodal area of the expansion chamber.

\section{Acknowledgments}

This work is supported by the National Natural Science Funds of China (51275275 and 50805088).

\section{References}

[1] J.G. Ih, B.H. Lee, Analysis of Higher-order Mode Effects in the Circular Expansion Chamber with Mean Flow, J. Acoust. Soc. Am. 77 (1985) 1377-1388.

[2] A. Selamet, Z.L. Ji, Acoustic Attenuation Performance of Circular Expansion Chambers with Offset Inlet/Outlet: I. Analytical Approach, J. Sound and Vib. 213 (1998): 601-617.

[3] Z. Fang, Z.L. Ji, Effect of Inlet and Outlet Locations on Acoustic Attenuation Characteristics of Elliptical and Oval Expansion Chambers, Noise and Vibration Control, 31(2011): 160-164.

[4] Z.G. Chu, F. Kuang, X.X. Gao, et al. Effects of Outlet Pipe Transition Circular Arc on Properties of Reactive Muffler and its Application, Transactions of the Chinese Society of Agricultural Engineering, 31(2015): 105-112.

[5] Z.X. Kang. A study on the Acoustic Attenuation Characteristics of Silencer and Perforated Elements [D]. Harbin Engineering University, 2009. 